\title{
Benzyl isothiocyanate suppresses IGF1R, FGFR3 and mTOR expression by upregulation of miR-99a-5p in human bladder cancer cells
}

\author{
JI-FAN LIN $^{1 *}$, TE-FU TSAI ${ }^{2,3 *}$, YI-CHIA LIN ${ }^{2,3}$, HUNG-EN CHEN $^{2}$, \\ KUANG-YU CHOU ${ }^{2,3}$ and THOMAS I-SHENG HWANG ${ }^{2-4}$
}

\begin{abstract}
${ }^{1}$ Central Laboratory, Shin-Kong Wu Ho-Su Memorial Hospital, Taipei 111; ${ }^{2}$ Division of Urology, School of Medicine, Fu-Jen Catholic University, New Taipei City $242 ;{ }^{3}$ Division of Urology, Department of Surgery, Shin-Kong Wu Ho-Su Memorial Hospital, Taipei 111; ${ }^{4}$ Department of Urology,

Taipei Medical University, Taipei 111, Taiwan, R.O.C.
\end{abstract}

Received May 29, 2018; Accepted November 26, 2018

DOI: 10.3892/ijo.2019.4763

\begin{abstract}
Benzyl isothiocyanate (BITC) is known for its pharmacological properties against malignant neoplasm, including bladder cancer (BC). The current study investigated microRNAs (miRNA or miR) expression profiles with an emphasis on the role of miR-99a-5p in BITC-treated BC cells. A quantitative polymerase chain reaction (qPCR) microarray containing 79 aberrantly expressed miRNAs in BC was used to detect miRNA expression in BITC-treated cells. Several dysregulated miRNAs were identified and further confirmed using miRNA stem-loop reverse transcription (RT)-qPCR in 5637 cells. Insulin-like growth factor 1 receptor (IGF1R), fibroblast growth factor receptor 3 (FGFR3) and mammalian target of rapamycin (mTOR) expression were determined by RT-qPCR and western blotting. Cell viability was evaluated using WST-1 reagent and apoptosis was monitored by determining the levels of cleaved-poly ADP-ribose polymerase and cleaved-caspase-3. BITC treatment significantly upregulated miR-99a-5p levels in a dose-dependent manner. miR-99a-5p overexpression decreased IGF1R, mTOR and FGFR 3 expression, predicted targets of miR-99a-5p. In addition, antisense miR-99a-5p sequences inhibited BITC-induced
\end{abstract}

Correspondence to: Dr Thomas I-Sheng Hwang, Division of Urology, Department of Surgery, Shin Kong Wu Ho-Su Memorial Hospital, 95 Wen Chang Road, Shih Lin, Taipei 111, Taiwan, R.O.C. E-mail: thomashwang0828@gmail.com

${ }^{*}$ Contributed equally

Abbreviations: BITC, benzyl isothiocyanate; $\mathrm{BC}$, bladder cancer; miRNAs, microRNAs; IGF1R, insulin-like growth factor 1 receptor; mTOR, mammalian target of rapamycin; FGFR3, fibroblast growth factor receptor 3

Key words: apoptosis, fibroblast growth factor receptor 3, insulin-like growth factor 1 receptor, microRNA-99a, mammalian target of rapamycin, urinary bladder neoplasms
miR-99a-5p overexpression, resulting in the restoration of protein expression and decreased cell viability. The current study identified multiple miRNAs responsive to BITC treatment, including miR-99a-5p. In addition, the induction of miR-99a-5p decreased IGF1R, mTOR and FGFR3 expression in BITC-treated BC cells. The current study provided novel insight into the antitumor mechanism by which BITC restores miR-99a-5p expression and decreases cancer cell survival.

\section{Introduction}

Bladder cancer (BC) is one of most common malignancies in urinary tract worldwide (1). A total of $~ 80 \%$ of patients with $\mathrm{BC}$ are diagnosed with non-muscle invasive BC (NMIBC) (2). The standard treatment for NMIBC is transurethral resection (TUR) (3). Recurrence of NMIBC following TUR is $60-80 \%$ (4). Recurrence is attributed to incomplete resection, growth of microscopic tumors, reimplantation of tumor cells or new tumor formation (5). Intravesical therapy with Bacillus Calmette-Geurin (BCG) vaccine or other chemotherapeutic agents, including mitomycin $\mathrm{C}$, are used to prevent or delay recurrence following TUR (6). However, $20-40 \%$ of patients respond poorly to these treatments (7) and new therapeutic modalities to prevent high recurrence rates are in a demand.

Benzyl isothiocyanate (BITC) is of the ITC family, which exerts anticancer activity by apoptosis induction in BC cells and inhibiting chemical-induced cancer in animal models (8). A recent study has revealed that BITC induces autophagic cell death in breast cancer (9). In addition, BITC treatment induces apoptosis and autophagy via inhibiting the mammalian target of rapamycin (mTOR) signaling pathway in prostate cancer cells (10). BITC has been reported to inhibit growth of pancreatic cancer cells through manipulating microRNA (miRNA or miR) expression. It is of interest to elucidate the mechanism detailing the anticancer effect of BITC in BC.

miRNAs are small noncoding RNAs ( $20-24$ nucleotides) that regulate target gene expression through translational blockage or mRNA degradation (11). Increasing studies have reported that miRNAs serve important roles in regulating tumor 
formation and progression (12). Numerous anticancer agents have been suggested to exert cell toxicity through manipulating miRNA expression (13). A recent study has reported that in patients with BC, miR-99a-5p is downregulated in cancerous tissues (14). The miR-99 family is known to be involved in the mTOR signaling pathway of other cancers $(15,16)$.

The present study focused on the association of miRNA with BITC-induced inhibitory effects in BC. It was hypothesized that BITC inhibited BC cell growth through altering the expression of certain miRNAs. miRNA expression profiles were explored in response to BITC treatment using a miRNA microarray approach. The target genes of miR-99a and downstream effectors were further investigated.

\section{Materials and methods}

Chemicals. All chemicals, unless otherwise stated, were purchased from Sigma-Aldrich (Merck KGaA, Darmstadt, Germany). BITC (purity, $98 \%$ ) was prepared as described previously (10).

Cell culture and transfection. Human BC cell lines RT4 (cat. no.HTB-2), 5637 (cat. no. HTB-9),HT1376 (cat. no. CRL-1472), HT1197 (cat. no. CRL-1473), T24 (cat. no. HTB-4) and humanureter-sumian-virus-40 transformed immortalized epithelial cell line SV-HUC-1 (cat. no. CRL-9520) were obtained from the American Type Culture Collection (Manassas, VA, USA). Cells were routinely checked for mycoplasma contamination using a polymerase chain reaction (PCR)-based method as described previously (17). RT4 cells were cultured in McCoy's 5A medium, HT1376 and HT1397 cells were maintained in Minimum essential medium, 5637 and T24 cells were cultured in RPMI-1640 and SV-HUC-1 cells were cultured in F12 medium; all media (Thermo Fisher Scientific, Inc., Waltham, MA, USA) were supplemented with $10 \%$ fetal bovine serum and essential supplements (Thermo Fisher Scientific, Inc.).

Cells at $70-80 \%$ confluence were transfected with below described plasmids in 6-well plates or $10-\mathrm{cm}$ dishes for $24 \mathrm{~h}$ prior to treatment with BITC $(20 \mu \mathrm{M})$ for $24 \mathrm{~h}$. Transfection of $1 \mu \mathrm{g} / \mathrm{ml}$ plasmid was performed using a polymer-based transfection reagent (Ultra293; GeneDireX, Inc., Taipei, Taiwan) according to the manufacturer's instructions and transfection efficiency was evaluated by reverse transcription-quantitative (RT-q) PCR.

Profiling of miRNAs expression using a BC miRNA RT-qPCR array. T24 bladder cancer cells were incubated with BITC $(20 \mu \mathrm{M})$ for $24 \mathrm{~h}$. The miProfile ${ }^{\mathrm{TM}}$ Human BC miRNA qPCR array (cat. no. QM-018; GeneCopoeia, Inc., Rockville, MD, USA), which is able to profile 79 aberrantly expressed miRNAs most relevant to $\mathrm{BC}$, was used to identify miRNA responses to BITC treatment of BC cells. Total RNA from control or BITC-treated 5637 cells was isolated using TRIzol reagent (Thermo Fisher Scientific, Inc.) according to the manufacturer's instructions, and the quality and concentration of RNA was determined using a NanoDrop2000 (Thermo Fisher Scientific, Inc.). miRNAs were reverse-transcribed $\left(37^{\circ} \mathrm{C} ; 60 \mathrm{~min}\right)$ from $2.5 \mu \mathrm{g}$ of total RNA using poly-A polymerase with an oligo(dT) adaptor from the All-in-One ${ }^{\mathrm{TM}}$ miRNA First Strand
cDNA Synthesis kit provided with the miRNA qPCR array (GeneCopoeia, Inc.). The qPCR array was performed in $20 \mu \mathrm{l}$ reactions containing $1 \mu \mathrm{l}$ RT product using the SYBR-Green (cat. no. KK4600; Kapa Biosystems; Roche Diagnostics, Indianapolis, IN, USA) detection on a StepOne Plus instrument (Thermo Fisher Scientific, Inc.). Primers for the miProfile ${ }^{\mathrm{TM}}$ human bladder cancer miRNA qPCR arrays were provided with the kit. The following protocol was used: $95^{\circ} \mathrm{C}$ for $3 \mathrm{~min}$; followed by 40 cycles of $95^{\circ} \mathrm{C}$ for $3 \mathrm{sec}$ and $60^{\circ} \mathrm{C}$ for $20 \mathrm{sec}$; melting curves were recorded between $60-95^{\circ} \mathrm{C}$ with using $0.1^{\circ} \mathrm{C} / \mathrm{sec}$ as a heat ramp and storage at $4^{\circ} \mathrm{C}$. Data was analyzed using All-in-One ${ }^{\mathrm{TM}}$ qPCR Primer Array Data Analysis software provided by GeneCopoeia, Inc. and the $2^{-\Delta \Delta C q}$ method was applied for quantification (18). Small nucleolar RNA U43 (SNORD43) was used as control.

Detection of miRNAs expression by stem-loop RT-qPCR. 5637 bladder cancer cells were incubated with BITC $(10 \mu \mathrm{M})$ for $24 \mathrm{~h}$. Total RNA was extracted and miR-99a-5p, miR-133b-5p, miR-30a-3p, miR-30a-5p, miR-125b-5p and miR-195-5p expression was determined by stem-loop RT-qPCR according to a previously published protocol (19). Stem-loop RT primers, universal reverse primer and miRNA specific forward primers are listed in Table I. miRNA was reverse transcribed into cDNA using the miRNA stem loop-RT primers and TaqMan ${ }^{\mathrm{TM}}$ MicroRNA Reverse Transcription kit (Thermo Fisher Scientific, Inc.). miRNAs quantification was performed using the StepOne Plus instrument (Thermo Fisher Scientific, Inc.), with universal reverse primer and miRNA specific forward primers. The Universal ProbeLibrary probe \#21 (UPL21) hydrolysis probe had the following sequence: $5^{\prime}-\mathrm{T}+\mathrm{G}+\mathrm{G}+\mathrm{C}+\mathrm{T}+\mathrm{C}+\mathrm{TG}-3$ ', where ' + ' identifies a unique nucleotide chemistry (Locked Nucleic Acid). SNORD43 was used as loading control. The following protocol was used: $95^{\circ} \mathrm{C}$ for $5 \mathrm{~min}$; followed by 40 cycles of $95^{\circ} \mathrm{C}$ for $5 \mathrm{sec}, 60^{\circ} \mathrm{C}$ for $10 \mathrm{sec}$ and $72^{\circ} \mathrm{C}$ for $1 \mathrm{sec}$ with $0.2^{\circ} \mathrm{C} / \mathrm{sec}$ heating and storage at $4^{\circ} \mathrm{C}$.

Construction of miR expression and reporter vectors. The miR-99a-5p expression vector (pSM-99a-5p) was constructed by annealing a paired oligonucleotides consisting of the mature miR-99a sequence (oligonucleotide 1, 5'-TGCTGAACCCGTA GATCCGATCTTGTGGTTTTGGCCACTGACTGACCACA AGATGATCTACGGGTT-3'; and oligonucleotide 2, 5'-CCT GAACCCGTAGATCATCTTGTGGTCAGTCAGTGGCCAA AACCACAAGATCGGATCTACGGGTTC-3') and cloned into a small-RNA expression vector (pSM; cat. no. 19170; Addgene, Inc., Cambridge, MA, USA) as previously described (20). The concept of a miRNA sponge targeting miRNA and attenuating its function has been well established $(21,22)$. Following a previous study describing the establishment of a let-7 sponge (23), the synthesized double strand oligonucleotides containing 3 repeats of matured miR-99a-5p antisense sequences were inserted to pmiR-GLO (Promega Corporation, Madison, WI, USA) generating a positive reporter construct (pmiR-GLO-99a-5p-PTS). 5637 and T24 cells ( $5 \times 10^{4}$ cells/well) at 7-80\% confluence were seeded into $24-w e l l$ plates and transfected with pmiR-GLO-99a-5p-PTS or empty control (pmiR-GLO; $1 \mu \mathrm{g} / \mathrm{ml}$ ) using a polymer-based transfection reagent (Ultra293; GeneDireX, Inc.) according to the manufacturer's instructions. Cells were treated with BITC $(20 \mu \mathrm{M})$ for $24 \mathrm{~h}$ post-transfection. Following 
Table I. Oligonucleotides and probe used for stem-loop RT-qPCR analysis.

A, Stem-Loop RT

\begin{tabular}{ll}
\hline Name & Sequence (5'-3') \\
\hline hsa-miR-99a-5p_RT & GTTGGCTCTGGTGCAGGGTCCGAGGTATTCGCACCAGAGCCAACCACAAG \\
hsa-miR-133b-5p_RT & GTTGGCTCTGGTGCAGGGTCCGAGGTATTCGCACCAGAGCCAACTAGCTG \\
hsa-miR-30a-3p_RT & GTTGGCTCTGGTGCAGGGTCCGAGGTATTCGCACCAGAGCCAACGCTGCA \\
hsa-miR-30a-5p_RT & GTTGGCTCTGGTGCAGGGTCCGAGGTATTCGCACCAGAGCCAACCTTCCA \\
hsa-miR-125b-5p_RT & GTTGGCTCTGGTGCAGGGTCCGAGGTATTCGCACCAGAGCCAACTGACAA \\
hsa-miR-195-5p_RT & GTTGGCTCTGGTGCAGGGTCCGAGGTATTCGCACCAGAGCCAACGCCAAT \\
SNORD43_RT & GTTGGCTCTGGTGCAGGGTCCGAGGTATTCGCACCAGAGCCAACAATCAG \\
\hline
\end{tabular}

B, qPCR

\begin{tabular}{ll}
\hline Name & Sequence (5'-3') \\
\hline hsa-miR-99a-5p_F & GTGAACCCGTAGATCCGAT \\
hsa-miR-133b-5p_F & GGGTTTGGTCCCCTTCAAC \\
hsa-miR-30a-3p_F & GTGCTTTCAGTCGGATGTT \\
hsa-miR-30a-5p_F & GGGTGTAAACATCCTCGAC \\
hsa-miR-125b-5p_F & GTGTCCCTGAGACCCTAAC \\
hsa-miR-195-5p_F & GGGGTAGCAGCACAGAAAT \\
SNORD43_F & GTGAACTTATTGACGGGCG \\
Universal reverse primer & GTGCAGGGTCCGAGGT
\end{tabular}

miR, micro RNA; RT, reverse transcription; qPCR, quantitative polymerase chain reaction; F, forward; SNORD43, small nucleolar RNA U43.

further 24 h, the activities of Firefly and Renilla luciferase were detected using Dual-luciferase kit (Promega Corporation). Relative protein levels were expressed as Firefly/Renilla luciferase.

Detection of IGF1R, FGFR3 and mTOR expression. T24 and 5637 bladder cancer cells seeded in 6-well plates (3x105 cells/well) were transfected with pSM-99a-5p, pmiR-GLO-99a-5p-PTS or control vectors $(1 \mu \mathrm{g} / \mathrm{ml})$ for $24 \mathrm{~h}$ using a polymer-based transfection reagent (Ultra293; GeneDireX, Inc.). Transfection efficiency was evaluated by RT-qPCR and luciferase activity assay as previously described (24). At $24 \mathrm{~h}$ post-transfection, transfected cells were incubated with BITC $(20 \mu \mathrm{M})$ for $24 \mathrm{~h}$. Cells were harvested and lysed by radioimmunoprecipitation assay lysis buffer (Sigma-Aldrich; Merck KGaA). Total proteins from BITC-treated cells transfected with pSM-99a-5p, pmiR-GLO-99a-5p-PTS or control vectors were collected from the lysate and subjected to the detection of insulin-like growth factor 1 receptor (1GF1R), fibroblast growth factor receptor 3 (FGFR3) and mTOR by western blot as described previously (10).

Cell viability assays and detection of apoptosis. Cell viability was determined in bladder cancer cells transfected with pSM-99a-5p, pmiR-GLO-99a-5p-PTS or control vectors treated with BITC $(20 \mu \mathrm{M})$ for $24 \mathrm{~h}$ using WST-1 reagent (Roche Diagnostics $\mathrm{GmbH}$, Mannheim, Germany) as previously described (25). The induction of apoptosis in BITC-treated cells was determined by assessment of cleaved (c-) poly ADP-ribose polymerase (PARP) and c-caspase-3 by western blotting.

Western blot analysis. Protein levels of cells treated with 10 or $20 \mu \mathrm{M}$ BITC for $24 \mathrm{~h}$ were examined using western blot analysis as described for the immunoblotting for IGF1R, FGFR3 and mTOR3. Antibodies against IGF1R (ab39675), FGFR3 (ab133644), mTOR (ab87540) were purchased from Abcam (Cambridge, UK). c-PARP (\#9532), c-caspase-3 (\#9661) and $\beta$-actin (\#4967) antibodies were purchased from Cell Signaling Technology (Danvers, MA, USA). Resolved proteins were transferred to polyvinyl difluoride membranes. Blots were blocked with $5 \%$ nonfat milk for $1 \mathrm{~h}$ at room temperature followed by incubation with primary antibodies $(1: 1,000)$ for $1 \mathrm{~h}$ at room temperature. Following washes with TBST (3x), blots were incubated with horseradish peroxidase-conjugated goat anti-rabbit $(1: 1,000$; cat. no. GTX213110-01) or anti-mouse secondary antibody (1:1,000; cat. no. GTX213111-01) (both from GeneTex, Inc., Irvine, CA, USA) for $1 \mathrm{~h}$ at room temperature followed by TBST washes $(3 \mathrm{x})$. Blots were visualized using an enhanced chemiluminescence detection system (Amersham; GE Healthcare, Chicago, IL, USA) according to the manufacturer's instruction. Densitometry was performed using Image J software $1.49 \mathrm{v}$ (National Institutes of Health, Bethesda, MD, USA). $\beta$-actin was used as internal control. Results are expressed as the mean \pm standard deviation (SD) of three independent experiments. 

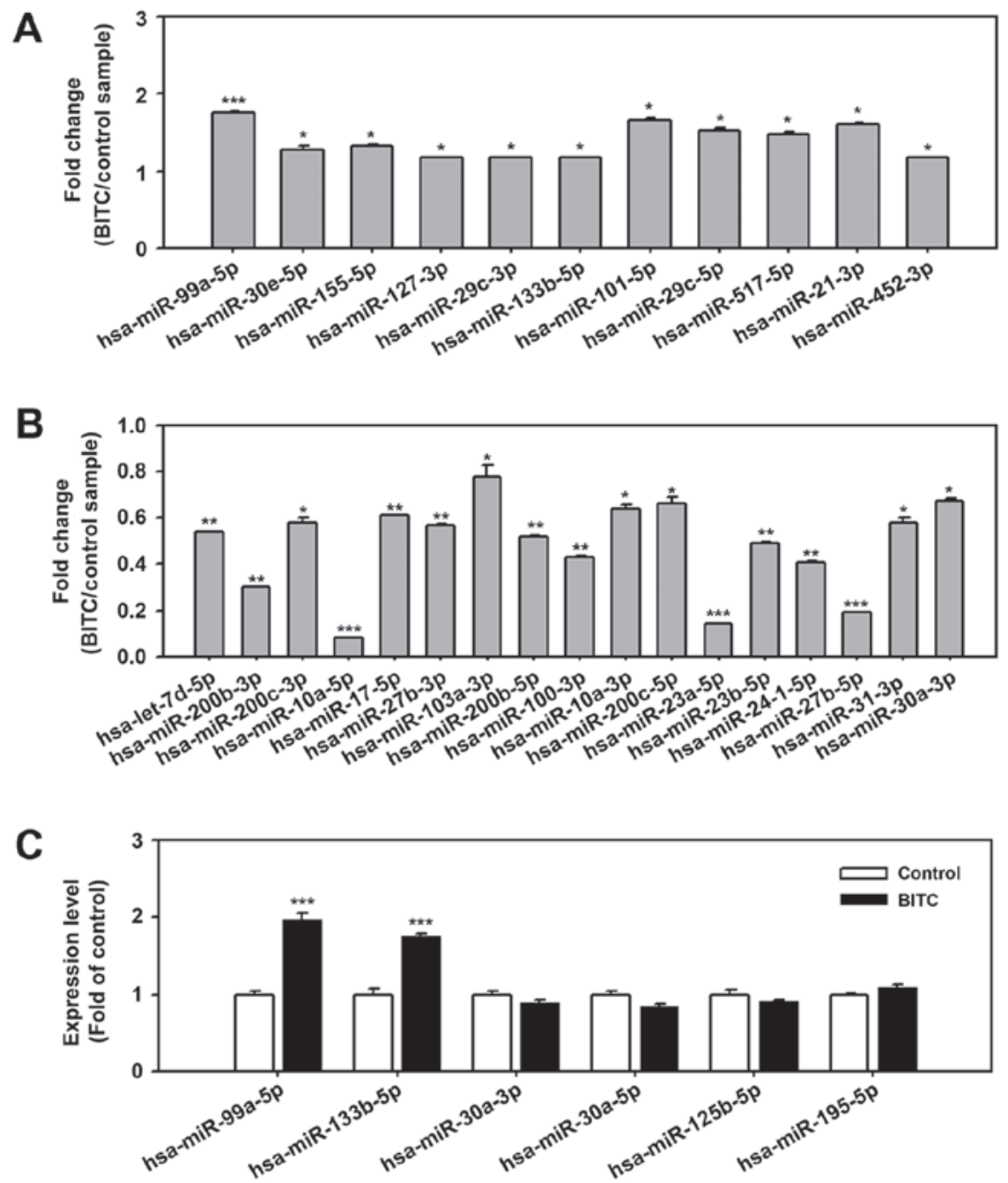

Figure 1. BITC treatment affects miR expression in 5637 cells. Expression levels of 79 bladder cancer-associated miRs were detected using a commercial miR qPCR array. Significantly (A) upregulated and (B) downregulated miRs identified in BITC-treated cells. (C) Expression of selected miRs detected by miR stem-loop reverse transcription-qPCR for validation. Expression changes were normalized to the control and are presented as fold change. The results are presented as the mean \pm standard deviation. ${ }^{\mathrm{P}} \mathrm{P}<0.05,{ }^{* * *} \mathrm{P}<0.01$ and ${ }^{* * * *} \mathrm{P}<0.001$ vs. control normalized to 1 (not presented). BITC, benzyl isothiocyanate; miR, microRNA; qPCR, quantitative polymerase chain reaction.

Statistical analysis. All experiments were performed $\geq 3$ times, each in triplicate and data are presented as the mean \pm SD. Statistical analysis between two samples were performed using Student's t-test. Multiple group comparisons were performed using one-way analysis of variance with Bonferroni's post-hoc tests. $\mathrm{P}<0.05$ was considered to indicate a statistically significant difference.

\section{Results}

BITC upregulates miR-99a-5p expression in BC. Dysregulation of miRNA has been reported in BC tissues, with 19 up- and 11 downregulated miRNAs (14). To investigate the effect on BITC treatment on miRNA expression in BC cells, expression profiles were determined using a miRNA qPCR array containing 79 aberrantly expressed miRNAs in BC. The results suggested that 11 miRNAs were significantly upregulated in BITC-treated BC cells compared with untreated control cells, including miR-30e-5p, miR-155-5p, miR-127-3p, miR-29c-3p, miR-133b-5p, miR-101-5p, miR-29c-5p, miR-517-5p, miR-21-3p, miR-452-3p (all $\mathrm{P}<0.05)$ and miR-99a-5p
$(\mathrm{P}<0.001$; Fig. 1A). A total of 17 miRNAs were significantly downregulated in BITC-treated 5637 cells compared with untreated control cells including miR-200c-3p, miR-103a-3p, miR-10a-3p, miR-200c-5p, miR-31-3p, miR-30a-3p (all $\mathrm{P}<0.05$ ), let-7d-5p, miR-200b-3p, miR-17-5p, miR-27b-3p, miR-200b-5p, miR-100-3p, miR-23b-5p, miR-24-1-5p (all $\mathrm{P}<0.01$ ), miR-10a-5p, miR-23a-5p and miR-27b-5p (all $\mathrm{P}<0.001$; Fig. 1B). BITC has been proposed to induce autophagy in breast cancer and prostate cancer cells $(9,10)$. To explore the correlation between dysregulated miRNAs and autophagy regulation, miRNAs reported to regulate the autophagy pathway, including miR-99a-5p (24), miR-133b-5p (26), miR-30a-3p (27), miR-30a-5p (28), miR-125b-5p (29) and miR-195-5p (30) were further validated by miRNA stem-loop RT-qPCR. Following validation by stem-loop RT-qPCR, data confirmed that miR-99a-5p and miR-133b-5p expression was significantly upregulated in 5637 cells treated with BITC compared with the normal control ( $\mathrm{P}<0.001$; Fig. 1C). However, expression of miR-30a-3p, miR-30a-5p, miR-125b-5p and miR-195-5p were not significantly affected by exposure to BITC. 

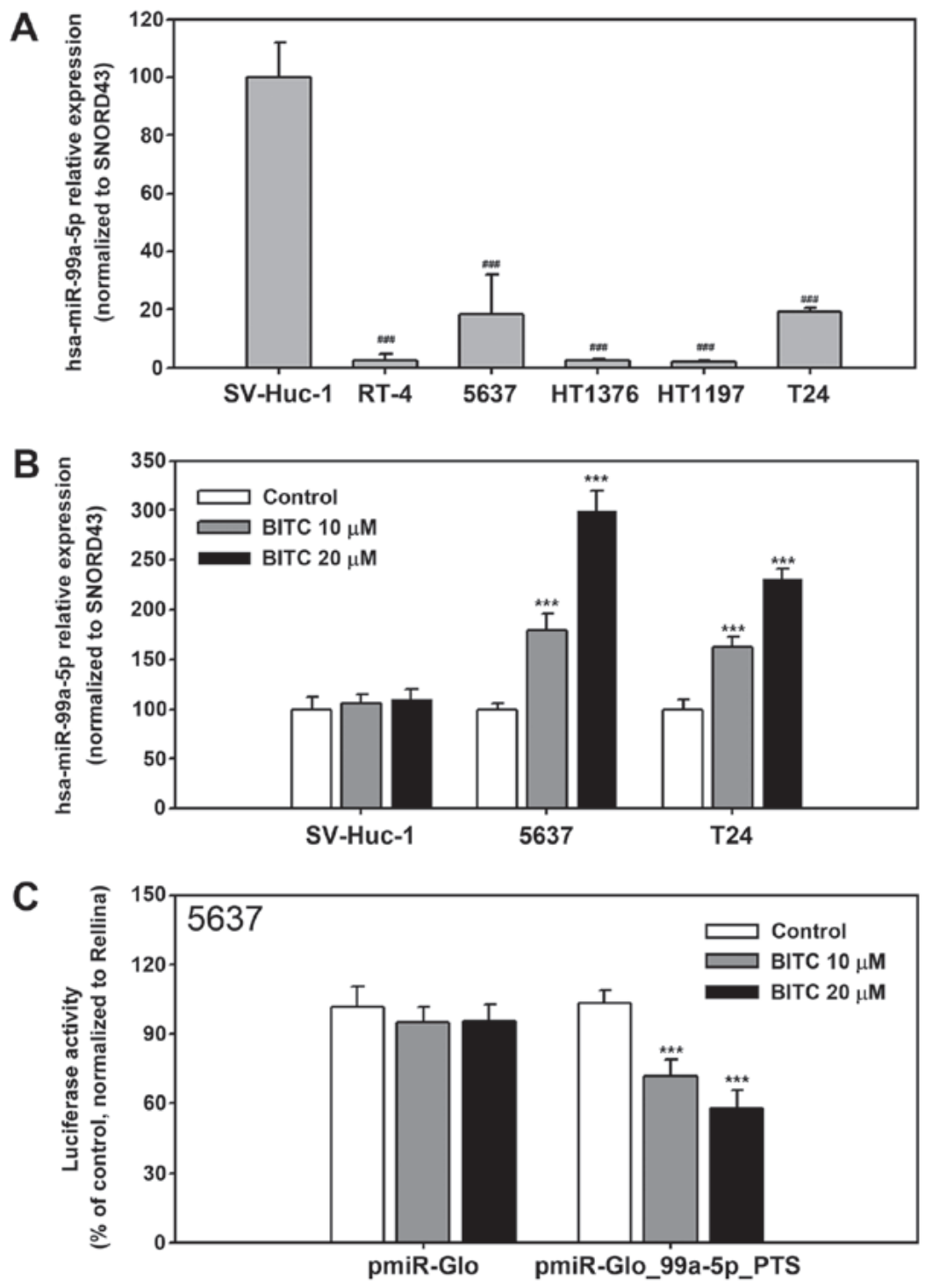

Figure 2. miR-99a-5p is upregulated in BITC-treated BC cells. (A) miR-99a-5p expression in human BC and immortalized urothelial cells

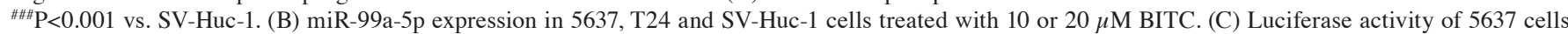
treated with 10 or $20 \mu \mathrm{M}$ BITC transfected with pmiR-Glo-99a-5p-PTS or empty vector. Results are presented as the mean \pm standard deviation. "P<0.05, ${ }^{* *} \mathrm{P}<0.01$ and ${ }^{* * *} \mathrm{P}<0.001$ vs. control. BITC, benzyl isothiocyanate; miR, microRNA; pmiR-Glo, empty luciferase vector; pmiR-Glo-99a-5p-PTS, vector containing miR-99a-5p antisense sequences.

To confirm that BITC induced miR-99a-5p expression, miR-99a-5p expression in untreated SV-Huc-1, RT4, 5637, HT1376, HT1197 and T24 cells was determined using stem-loop miRNA RT-qPCR. As presented in Fig. 2A, miR-99a-5p expression was significantly downregulated in all BC cells compared with the SV-Huc-1 normal cells $(\mathrm{P}<0.001)$. 5637 and T24 cells were used in following based on higher transfection efficiencies compared with the other cell lines. BITC treatment increased miR-99a-5p expression in 5637 and T24 cells compared with the untreated cells $(\mathrm{P}<0.001)$; no significant changes were observed for SV-Huc-1 cells (P>0.05; Fig. 2B). To further evaluate the effect of BITC treatment on miR-99a-5p expression, luciferase assays were performed using pmiR-Glo-99a-5p-PTS, which is able to bind miR-99a-5p. The reporter construct was transfected into 5637 cells and cells were treated with BITC for $24 \mathrm{~h}$ post transfection. The results demonstrated a significant dose-dependent decrease in luciferase activity upon BITC treatment compared with the untreated control, indicating miR-99a-5p upregulation in BITC-treated cells ( $\mathrm{P}<0.001$; Fig. 2C).

miR-99a-5p overexpression and BITC treatment decrease IGFIR, FGFR 3 and mTOR expression in BC cells. Changes in the expression of target genes of miR-99a-5p were evaluated following miR-9a-5p overexpression and BITC treatment of BC cells. IGF1R, mTOR and FGFR3 expression was determined in 5637 and T24 cells transfected with a miR-99a-5p overexpressing vector. IGF1R, mTOR and FGFR3 mRNA was significantly decreased in pSM-99a-5p transfected 5637 and T24 cells compared with the empty vector control $(\mathrm{P}<0.01, \mathrm{P}<0.001$ and $\mathrm{P}<0.001$, respectively; Fig. $3 \mathrm{~A})$. Protein expression levels were also significantly decreased in the miR-99a-5p overexpression samples compared with the control $(\mathrm{P}<0.001$ for 5637 cells and $\mathrm{P}<0.01, \mathrm{P}<0.001$ and $\mathrm{P}<0.01$ for IGF1R, mTOR and FGFR3 in T24, respectively; Fig. 3B). To investigate the expression of IGF1R, mTOR and FGFR3 

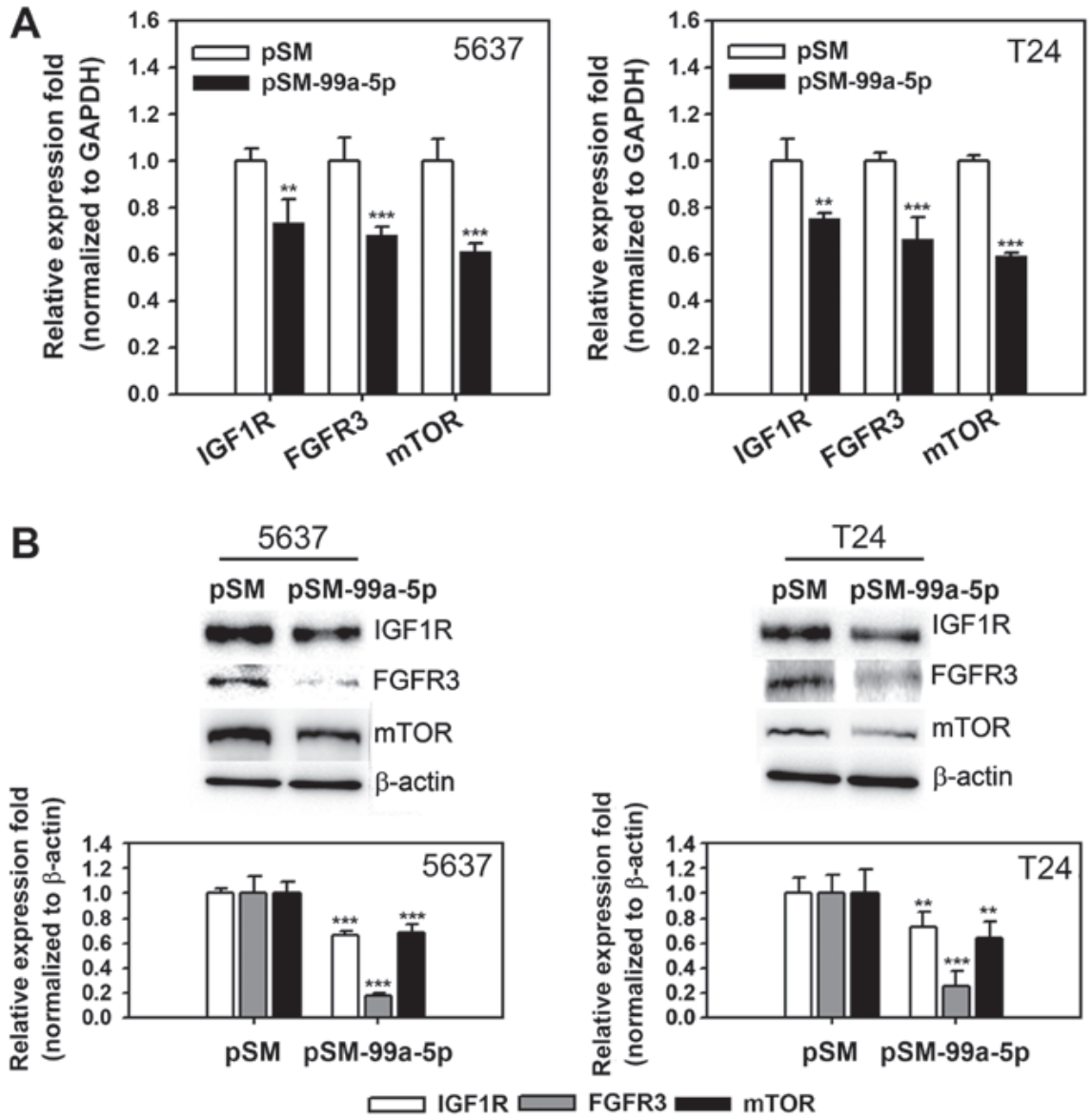

Figure 3. miR-99a-5p overexpression decreases IGF1R, mTOR and FGFR3 expression. Bladder cancer 5637 and T24 cells were transfected with pSM-99a-5p or an empty vector control and (A) mRNA and (B) protein expression of IGF1R, mTOR and FGFR3 were detected by reverse transcription-quantitative polymerase chain reaction and western blot assays, respectively. $\beta$-actin was used as internal control. Results are representative of three independent experiments and are presented as the mean \pm standard deviation. ${ }^{*} \mathrm{P}<0.05,{ }^{* * *} \mathrm{P}<0.01$ and ${ }^{* * * *} \mathrm{P}<0.001$ vs. pSM. miR, microRNA; IGF1R, insulin-like growth factor 1 receptor; mTOR, mammalian target of rapamycin; FGFR3, fibroblast growth factor receptor 3.

in BITC-treated cells, mRNA and protein expression was determined in 5637 and T24 cells treated with BITC. As presented in Fig. 4A, BITC treatment $(20 \mu \mathrm{M})$ resulted in decreased mRNA expression of IGF1R, mTOR and FGFR3 compared with the untreated control $(\mathrm{P}<0.001$ for 5637 and $\mathrm{P}<0.001, \mathrm{P}<0.01$ and $\mathrm{P}<0.001$ for IGF1R, mTOR, and FGFR3 in T24, respectively). Protein expression was significantly decreased in BITC-treated cells in a dose-dependent manner compared with the untreated control $(\mathrm{P}<0.01$ and $\mathrm{P}<0.001$ for 10 and $20 \mu \mathrm{M}$ BITC, respectively; Fig. 4B). The results indicated that miR-99a-5p overexpression and BITC treatment inhibited the expression of IGF1R, mTOR and FGFR3 prosurvival proteins in $\mathrm{BC}$ cells.

miR-99a-5p inhibition attenuates BITC-induced IGFIR, FGFR3 and mTOR downregulation and decreased cell viability. A previous study by the authors focused on pmiR-Glo-99a-5p-PTS, which expressed antisense miR-99a-5p and exhibited inhibitory effects on miR-99a-5p function (24). To confirm that IGF1R, mTOR and FGFR3 inhibition was mediated by miR-99a-5p upregulation through BITC treatment, experiments using pmiR-Glo-99a-5p-PTS acting as competitors to BITC-induced miR-99a-5p expression were performed. IGF1R, mTOR and FGFR3 expression was detected in transfected cells that received $24 \mathrm{~h}$ BITC treatment
$(20 \mu \mathrm{M})$. As presented in Fig. 5A, overexpression of miR-99a-5p induced IGF1R and mTOR expression in 5637 cell $(\mathrm{P}<0.001$ and $\mathrm{P}<0.01$, respectively). Furthermore, IGF1R, mTOR and FGFR3 protein expression downregulation in BITC-treated cells was significantly reversed by antisense miR-99a-5p expressing, BITC-treated cells $(\mathrm{P}<0.001, \mathrm{P}<0.05$ and $\mathrm{P}<0.001$, respectively). Effects of miR-99a-5p inhibition on the viability of BITC-treated cells were further evaluated. Cell viability was significantly decreased in BITC-treated cells compared with the untreated controls $(\mathrm{P}<0.001$; Fig. 5B). Inhibition of miR-99a-5p significantly reversed the BITC-induced viability decrease in 5637 and $\mathrm{T} 24$ cells $(\mathrm{P}<0.01$ and $\mathrm{P}<0.05$, respectively; Fig. 5B). The results suggested that BITC treatment suppressed IGF1R, mTOR and FGFR3 expression by upregulating miR-99a-5p levels. However, there may be further effectors, in addition to miR-99a-5p, that contributed to BITC-induced cytotoxicity in BC cells.

Effects of miR-99a-5p overexpression are enhanced by BITC treatment in $B C$ cells. It was evaluated if combination of miR-99a-5p overexpression and BITC treatment enhanced cell death in BC cells compared with the single treatments. As presented in Fig. 6A, BITC treatment $(10 \mu \mathrm{M})$ and miR-99a-5p overexpression alone significantly decreased cell viability in 5637 and T24 cells compared with the untreated cells $(\mathrm{P}<0.001)$. 

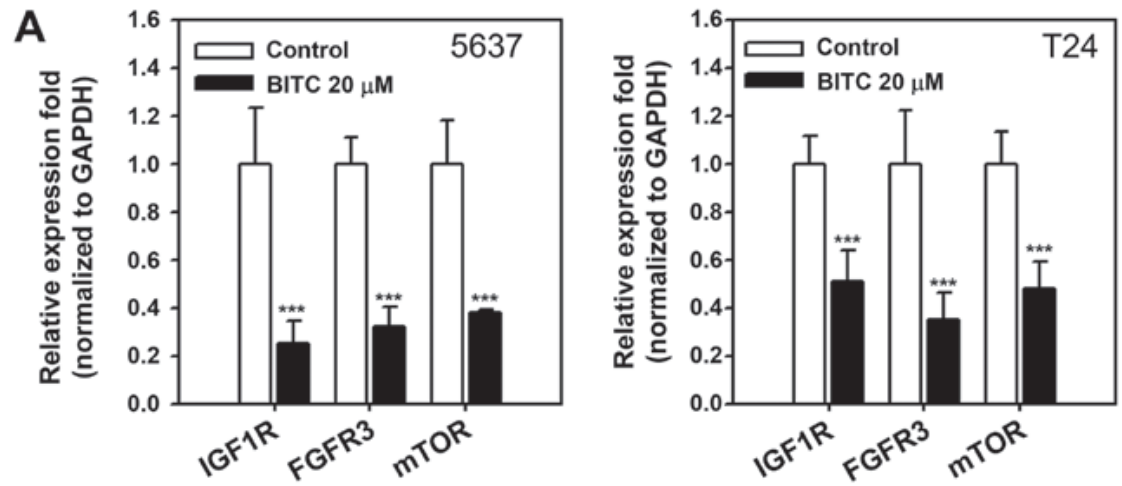

B
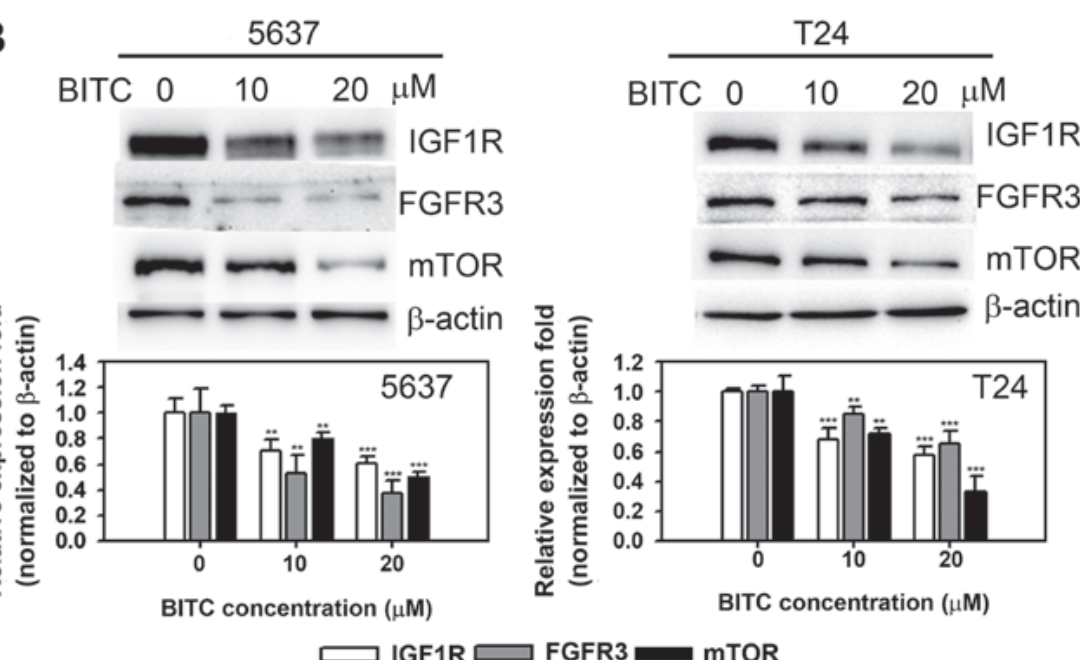

Figure 4. BITC treatment decreases IGF1R, mTOR, and FGFR3 expression. (A) IGF1R, mTOR and FGFR3 mRNA expression in BITC-treated (20 $\mu$ M) 5637 and T2 4 cells determined by reverse transcription-quantitative polymerase chain reaction. (B) IGF1R, mTOR and FGFR3 protein expression in BITC-treated (10 and $20 \mu \mathrm{M}) 5637$ and T24 cells determined by western blotting. $\beta$-actin was used as internal control. Results are representative of three independent experiments and are presented as the mean \pm standard deviation. ${ }^{*} \mathrm{P}<0.05,{ }^{* *} \mathrm{P}<0.01$ and ${ }^{* * *} \mathrm{P}<0.001$ vs. control. BITC, benzyl isothiocyanate; IGF1R, insulin-like growth factor 1 receptor; mTOR, mammalian target of rapamycin; FGFR3, fibroblast growth factor receptor 3.

Cell viability was further significantly decreased when combining the two treatments in 6537 and T24 cells compared with miR-99a-5p overexpression alone $(\mathrm{P}<0.001)$. Apoptosis induction was evaluated by determining the cleavage of PARP and caspase-3.Cleaved protein levels were significantly increased miR-99a-5p overexpressing or BITC-treated cells compared with the untreated cells $(\mathrm{P}<0.01$ and $\mathrm{P}<0.001$, respectively; Fig. 6B). The combination of miR-99a-5p overexpression and BITC treatment further significantly increased the c-PARP and c-caspase-3 levels compared with the BITC treatment group $(\mathrm{P}<0.001)$. The results suggested that miR-99a-5p overexpression enhances the effects of BITC in BC cells.

\section{Discussion}

$\mathrm{BC}$ is one of the leading causes of cancer-associated mortality worldwide (31). Novel therapeutic approaches preventing the recurrence or improving the survival of patients with $\mathrm{BC}$ are desirable. In the current study, it was described that BITC inhibited IGF1R, mTOR and FGFR3 expression through upregulation of the tumor suppressing miR-99a-5p. The results further demonstrated that elevation of miR-99a-5p levels enhanced BITC-induced cytotoxicity in BC cells. Increasing evidence has highlighted anti-cancer activities of BITC in a variety of tumor cell lines and in rodent animal models (32).
BITC is suggested to inhibit growth, induce apoptosis and G2/M phase cell cycle arrest in BC cells (33). The current study suggested that BITC decreased 5637 and T24 cell viability by induction of apoptosis.

miRNAs are important regulatory components in tumorigenesis and several miRNAs are considered as therapeutic targets in BC (34). Tumor suppressing activities of miR-99a-5p have been investigated in various types of cancer; miR-99a-5p exerts anti-metastasis abilities in human non-small cell lung cancer cells by inhibiting protein kinase B1 and in oral cancer by inhibiting myotubularin-related protein 3 expression $(35,36)$. In mammary gland cells miR-99a-5p modulates transforming growth factor- $\beta$ induced epithelial to mesenchymal plasticity (37). mTOR targeting and inhibition of cell proliferation or induction of apoptosis have been demonstrated in anaplastic thyroid (15), breast (16) and cervical cancer (38). miR-99a-5p controls IGF1R and mTOR expression in human hepatocellular carcinoma (39-41) and has been reported to be downregulated in human $\mathrm{BC}$, leading to the upregulation of FGFR3 $(14,42)$. IGF1R, FGFR3 and mTOR are known anti-apoptotic regulators (39-41). The results of the current study indicated antitumor effects of miR-99a-5p by inducing apoptosis in BC. A previous study reported that miR-99a-5p is downregulated in bladder urothelial carcinoma tissue compared with normal tissue (14). 

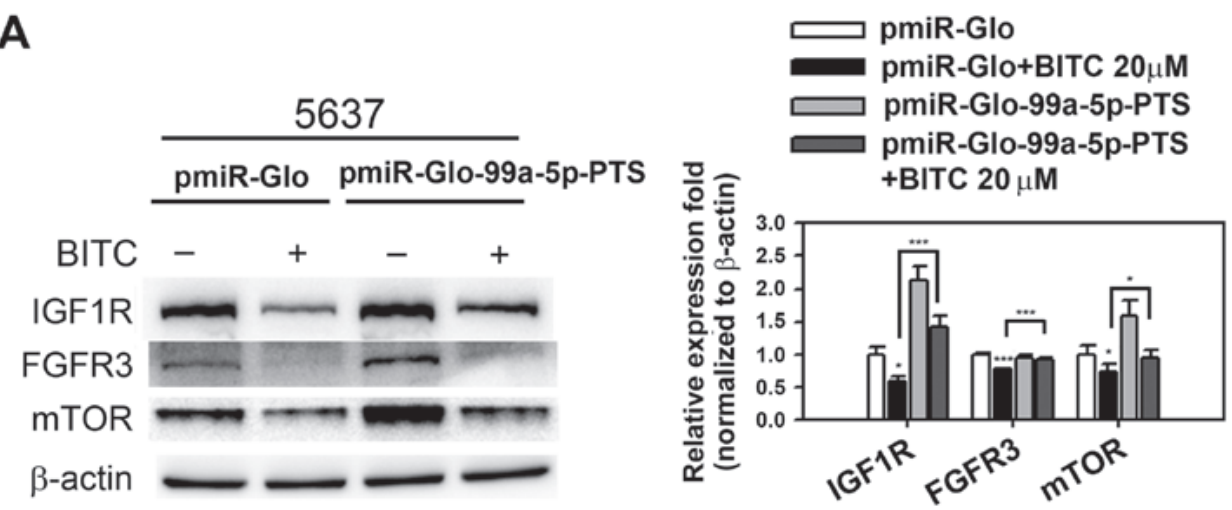

B

5637
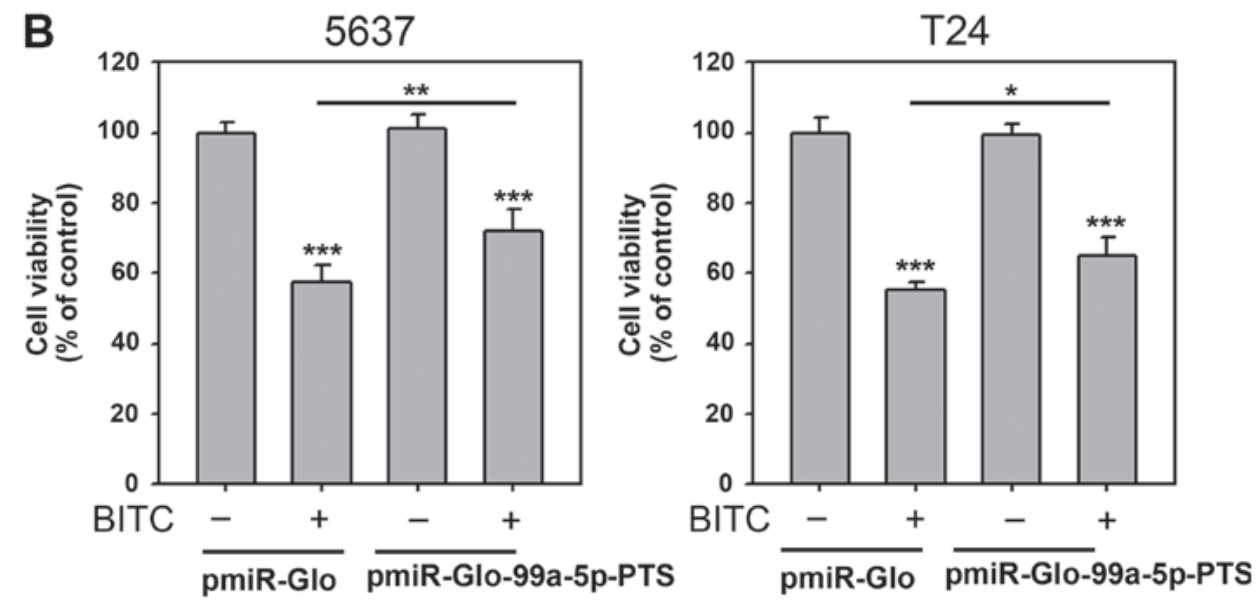

Figure 5. Inhibition of BITC-induced miR-99a-5p expression attenuates protein expression and decreases cell viability. Bladder cancer cells transfected with pmiR-Glo-99a-5p-PTS or empty vector were treated with BITC (20 $\mu \mathrm{M})$. (A) IGF1R, mTOR and FGFR3 protein expression in 5637 cells treated as detailed above determined by western blotting. $\beta$-actin was used as internal control. Results are representative of three independent experiments. (B) Cell viability of 5637 and T24 cells treated as indicated was determined using WST-1 reagent. Results are presented as the mean \pm standard deviation. ${ }^{*}<0.05$, ${ }^{* *} \mathrm{P}<0.01$ and ${ }^{* * *} \mathrm{P}<0.001$ vs. pmiR-Glo. BITC, benzyl isothiocyanate; miR, microRNA; pmiR-Glo, empty luciferase vector; pmiR-Glo-99a-5p-PTS, vector containing miR-99a-5p antisense sequences; IGF1R, insulin-like growth factor 1 receptor; mTOR, mammalian target of rapamycin; FGFR3, fibroblast growth factor receptor 3 .

Furthermore, expression levels and prognostic roles of IGF-1R, mTOR and FGFR3 have previously been reported for BC (43-45). The small number of patients with BC that participated in the current study describes a limitation and IGF1R, mTOR and FGFR3 expression will be investigated further in future experiments.

miR-99a-5p exhibits anticancer activity in various cancer types and BITC has been reported to induce apoptosis in BC cells (46). The current study demonstrated that BITC induced miR-99a-5p expression in BC cells but not normal human urothelial cells. Furthermore, it was suggested that miR-99a-5p may be involved in the regulation of IGF1R, mTOR and FGFR3 in BITC-treated BC cells. A previous review has reported the application of a miRNA sponge, containing multiple miRNA binding sites, in miRNA inhibition $(21,22)$. The results of the current study confirmed that overexpression of miR-99a-5p sponge reversed IGF1R, mTOR and FGFR3 protein expression downregulation in BITC-treated cells. A previous review has addressed the antitumor mechanisms exerted by various ITCs (47). The report proposed that production of reactive oxygen species (ROS) is the common link of ITCs in apoptosis induction. In addition, normal cells exhibit increasing resistance to ROS production and apoptosis induced by
ITCs, suggesting that the induction of miR-99a-5p by BITC treatment in $\mathrm{BC}$ cells may contribute to ROS production. These suggestions require to be verified in future experiments.

Various miRNAs that promote cancer cell death are recognized as potential novel anti-cancer agents in various cancers, including BC (34). miR-34a is downregulated during cancer progression and considered a novel target for treating various types of cancer (48). miRNAs rapidly degrade in circulating blood, making an oral or intravenous administration ideal for delivery (49). Bladder instillation had been routinely performed in clinic using chemotherapeutic agents, including $\mathrm{BCG}$ or mitomycin $\mathrm{C}$ to prevent recurrence of BC (50). Intravesical therapy by delivery of small non-coding RNA is an alternative approach to overcome drug delivery system problems and successfully deliver siRNAs in vivo (51). Therapeutic effects of miR-582-5p and $-3 p$ (52) and miR-145 (53) administered intravesically in a mouse orthotopic model suggest promising effects against BC. N-acetylcysteine (NAC)-conjugated BITC is the major metabolite detected in urine collected from human donors in different studies (54). It remains to be investigated whether NAC-BITC has the ability to induce miR-99a-5p expression. The present study focused on the dysregulation of miRNAs 

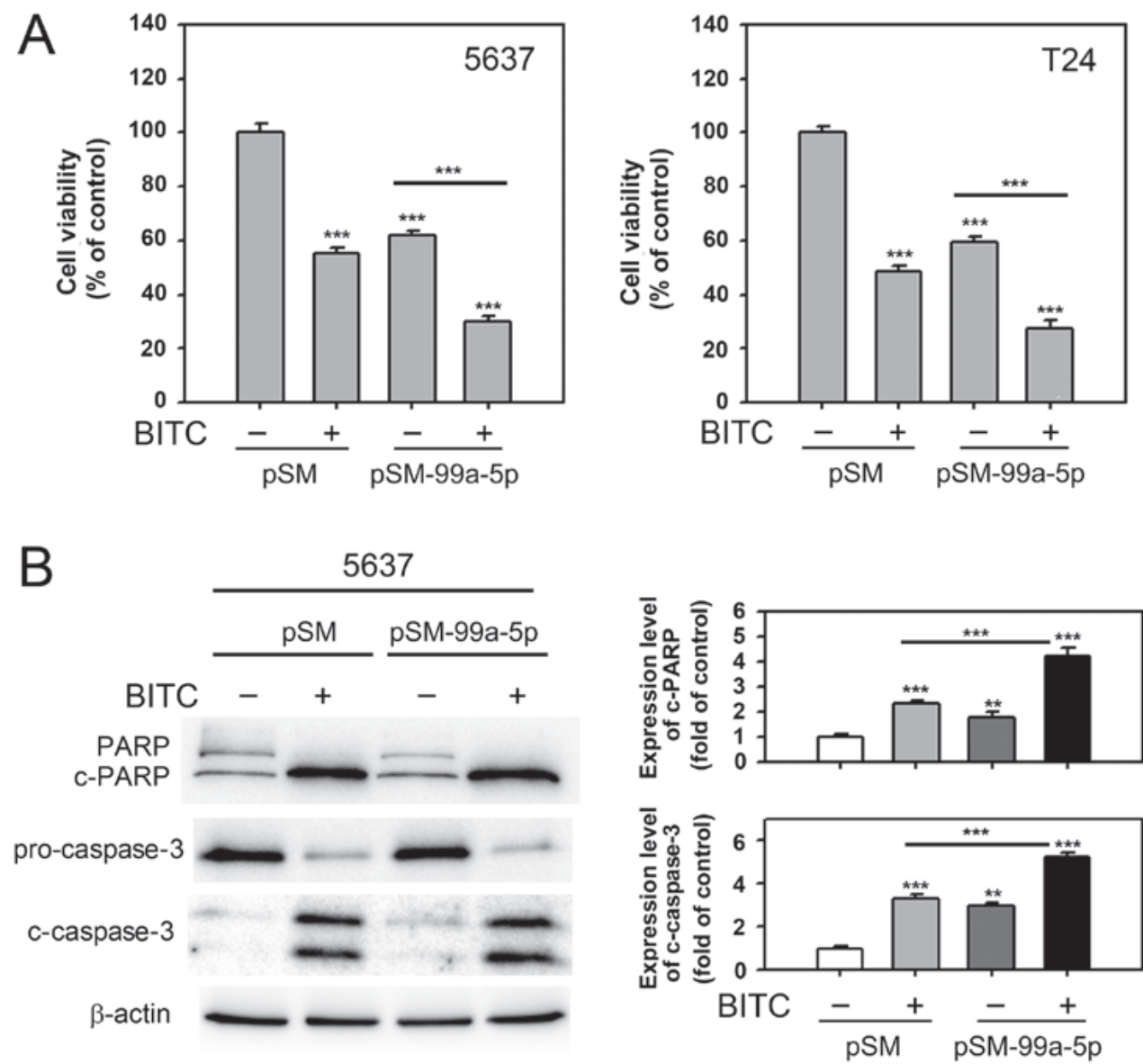

Figure 6. miR-99a-5p overexpression enhances BITC-induced cytotoxicity and apoptosis. Bladder cancer cells transfected with pSM-99a-5p or empty vector were treated with BITC $(20 \mu \mathrm{M})$. (A) Cell viability of 5637 and T24 cells with indicated treatment detected by WST-1. (B) Levels of pro-apoptotic marker proteins, c-PARP, PARP, pro-Casp3 and c-Casp3 were detected in 5637 cells with indicated treatment using western blotting. $\beta$-actin was used as internal control. Results are representative of three independent experiments and are presented as the mean \pm standard deviation. ${ }^{*} \mathrm{P}<0.05,{ }^{* *} \mathrm{P}<0.01$ and ${ }^{* * * *} \mathrm{P}<0.001$ vs. pSM. BITC, benzyl isothiocyanate; miR, microRNA; c-, cleaved; PARP, poly ADP-ribose polymerase.

following BITC treatment in BC. In initial experiments, miR-99a-5p demonstrated the strongest response to BITC treatment and was selected for further investigation of its role in $\mathrm{BC}$ progression. It was demonstrated that ectopic miR-99a-5p expression in combination with BITC treatment decreased cell viability of BC cells compared with either single treatment. The current study suggested that miR-99a-5p may be a novel anticancer agent, alone or combined with other chemotherapeutic agents, and has the potential to inspire future experiments in a preclinical setting.

In this study, it was demonstrated for the first time that BITC treatment inhibited expression of prosurvival proteins IGF1R, mTOR and FGFR3 by upregulation of miR-99a-5p in human BC cells. miR-99a-5p overexpression potentiated the cytotoxicity of BITC in BC cells. Orthotopic animal models using in vivo imaging system detection have been widely applied in BC studies (55) and miRNA replacement therapy provides strong preclinical evidence for miRNA-based treatment of cancer $(56,57)$. These preclinical results may shape future experiments studying the effects of miR-99a-5p in BC treatment.

\section{Acknowledgements}

Not applicable.

\section{Funding}

This study was supported by Ministry of Science and Technology, Taiwan (grant no. NSC102-2314-B-341-003-MY3) and Shin Kong Wu Ho-Su Memorial Hospital (grant nos. SKH-8302-103-DR-13, SKH-8302-103-NDR-06, SKH-8302-104-0201 and SKH-8302-104-0202).

\section{Availability of data and material}

The datasets analyzed during the current study are available from the corresponding author on reasonable request.

\section{Authors' contributions}

JFL and TFT conceived and performed the experiments, data interpretation and writing of the manuscript. JFL and TIH designed the study. YCL, HEC and KYC provided the study materials and participate in the interpretation of the experiment data. All authors read and approved the final version of the manuscript.

\section{Ethics approval and consent to participate}

Not applicable. 


\section{Patient consent for publication}

Not applicable.

\section{Competing interests}

The authors declare that they have no competing interests.

\section{References}

1. Siegel R, Naishadham D and Jemal A: Cancer statistics, 2012. CA Cancer J Clin 62: 10-29, 2012.

2. Chavan S, Bray F, Lortet-Tieulent J, Goodman M and Jemal A: International variations in bladder cancer incidence and mortality. Eur Urol 66: 59-73, 2014.

3. Gudjónsson S, Adell L, Merdasa F, Olsson R, Larsson B, Davidsson T, Richthoff J, Hagberg G, Grabe M, Bendahl PO, et al: Should all patients with non-muscle-invasive bladder cancer receive early intravesical chemotherapy after transurethral resection? The results of a prospective randomised multicentre study. Eur Urol 55: 773-780, 2009.

4. Metts MC, Metts JC, Milito SJ and Thomas CR Jr: Bladder cancer: A review of diagnosis and management. J Natl Med Assoc 92: 285-294, 2000.

5. Kaufman DS, Shipley WU and Feldman AS: Bladder cancer. Lancet 374: 239-249, 2009.

6. Brausi M, Witjes JA, Lamm D, Persad R, Palou J, Colombel M, Buckley R, Soloway M, Akaza $H$ and Böhle A: A review of current guidelines and best practice recommendations for the management of nonmuscle invasive bladder cancer by the International Bladder Cancer Group. J Urol 186: 2158-2167, 2011.

7. Brausi M, Oddens J, Sylvester R, Bono A, van de Beek C, van Andel G, Gontero P, Turkeri L, Marreaud S, Collette S, et al Side effects of Bacillus Calmette-Guérin (BCG) in the treatment of intermediate- and high-risk Ta, T1 papillary carcinoma of the bladder: Results of the EORTC genito-urinary cancers group randomised phase 3 study comparing one-third dose with full dose and 1 year with 3 years of maintenance BCG. Eur Urol 65 69-76, 2014.

8. Smith TJ: Mechanisms of carcinogenesis inhibition by isothiocyanates. Expert Opin Investig Drugs 10: 2167-2174, 2001.

9. Xiao D, Bommareddy A, Kim SH, Sehrawat A, Hahm ER and Singh SV: Benzyl isothiocyanate causes FoxO1-mediated autophagic death in human breast cancer cells. PLoS One 7 : e32597, 2012

10. Lin JF, Tsai TF, Liao PC, Lin YH, Lin YC, Chen HE, Chou KY and Hwang TI: Benzyl isothiocyanate induces protective autophagy in human prostate cancer cells via inhibition of mTOR signaling. Carcinogenesis 34: 406-414, 2013.

11. He L and Hannon GJ: MicroRNAs: Small RNAs with a big role in gene regulation. Nat Rev Genet 5: 522-531, 2004.

12. Iorio MV and Croce CM: Causes and consequences of microRNA dysregulation. Cancer J 18: 215-222, 2012.

13. Phuah NH and Nagoor NH: Regulation of microRNAs by natura agents: New strategies in cancer therapies. BioMed Res Int 2014: $804510,2014$.

14. Tsai TF, Lin YC, Chen HE, Chou KY, Lin JF and Hwang TI: Involvement of the insulin-like growth factor I receptor and its downstream antiapoptotic signaling pathway is revealed by dysregulated microRNAs in bladder carcinoma. Urol Sci 25: 58-64, 2014

15. Huang HG, Luo X, Wu S and Jian B: MiR-99a inhibits cell proliferation and tumorigenesis through targeting mTOR in human anaplastic thyroid cancer. Asian Pac J Cancer Prev 16: 4937-4944, 2015

16. Yang Z, Han Y, Cheng K, Zhang G and Wang X: miR-99a directly targets the mTOR signalling pathway in breast cancer side population cells. Cell Prolif 47: 587-595, 2014.

17. Lin JF, Lin YC, Lin YH, Tsai TF, Chou KY, Chen HE and Hwang TI: Zoledronic acid induces autophagic cell death in human prostate cancer cells. J Urol 185: 1490-1496, 2011.

18. Livak KJ and Schmittgen TD: Analysis of relative gene expression data using real-time quantitative PCR and the 2(-Delta Delta C(T)) method. Methods 25: 402-408, 2001.

19. Varkonyi-Gasic E, Wu R, Wood M, Walton EF and Hellens RP: Protocol: A highly sensitive RT-PCR method for detection and quantification of microRNAs. Plant Methods 3: 12, 2007.
20. Lin CJ, Gong HY, Tseng HC, Wang WL and Wu JL: miR-122 targets an anti-apoptotic gene, Bcl-w, in human hepatocellular carcinoma cell lines. Biochem Biophys Res Commun 375: 315-320, 2008

21. Ebert MS, Neilson JR and Sharp PA: MicroRNA sponges: Competitive inhibitors of small RNAs in mammalian cells. Nat Methods 4: 721-726, 2007.

22. Ebert MS and Sharp PA: MicroRNA sponges: Progress and possibilities. RNA 16: 2043-2050, 2010.

23. Deng L, Yang SB, Xu FF and Zhang JH: Long noncoding RNA CCAT1 promotes hepatocellular carcinoma progression by functioning as let-7 sponge. J Exp Clin Cancer Res 34: 18, 2015.

24. Tsai TF, Lin JF, Chou KY, Lin YC, Chen HE and Hwang TI: miR-99a-5p acts as tumor suppressor via targeting to mTOR and enhances RAD001-induced apoptosis in human urinary bladder urothelial carcinoma cells. OncoTargets Ther 11: 239-252, 2018.

25. Lin YC, Lin JF, Wen SI, Yang SC, Tsai TF, Chen HE, Chou KY and Hwang TI: Inhibition of high basal level of autophagy induces apoptosis in human bladder cancer cells. J Urol 195 1126-1135, 2016.

26. Sugiyama T, Taniguchi K, Matsuhashi N, Tajirika T, Futamura M, Takai T, Akao Y and Yoshida K: MiR-133b inhibits growth of human gastric cancer cells by silencing pyruvate kinase muscle-splicer polypyrimidine tract-binding protein 1 . Cancer Sci 107: 1767-1775, 2016.

27. Zhang L, Cheng R and Huang Y: MiR-30a inhibits BECN1-mediated autophagy in diabetic cataract. Oncotarget 8: 77360-77368, 2017.

28. Fu XT, Shi YH, Zhou J, Peng YF, Liu WR, Shi GM, Gao Q, Wang XY, Song K, Fan J, et al: MicroRNA-30a suppresses autophagy-mediated anoikis resistance and metastasis in hepatocellular carcinoma. Cancer Lett 412: 108-117, 2018.

29. Wang S, Wu J, Ren J, Vlantis AC, Li MY, Liu SY, Ng EK, Chan AB, Luo DC, Liu Z, et al: MicroRNA-125b Interacts with Foxp3 to Induce Autophagy in Thyroid Cancer. Mol Ther 26: 2295-2303, 2018.

30. Shi G, Shi J, Liu K, Liu N, Wang Y, Fu Z, Ding J, Jia L and Yuan W: Increased miR-195 aggravates neuropathic pain by inhibiting autophagy following peripheral nerve injury. Glia 61: 504-512, 2013

31. Siegel RL, Miller KD and Jemal A: Cancer statistics, 2015. CA Cancer J Clin 65: 5-29, 2015.

32. Rao CV: Benzyl isothiocyanate: Double trouble for breast cancer cells. Cancer Prev Res (Phila) 6: 760-763, 2013.

33. Tang $L$ and Zhang Y: Dietary isothiocyanates inhibit the growth of human bladder carcinoma cells. J Nutr 134: 2004-2010, 2004

34. Catto JW, Alcaraz A, Bjartell AS, De Vere White R, Evans CP, Fussel S, Hamdy FC, Kallioniemi O, Mengual L, Schlomm T, et al: MicroRNA in prostate, bladder, and kidney cancer: A systematic review. Eur Urol 59: 671-681, 2011

35. Yu SH, Zhang CL, Dong FS and Zhang YM: miR-99a suppresses the metastasis of human non-small cell lung cancer cells by targeting AKT1 signaling pathway. J Cell Biochem 116: 268-276, 2015.

36. Kuo YZ, Tai YH, Lo HI, Chen YL, Cheng HC, Fang WY, Lin SH, Yang CL, Tsai ST and Wu LW: MiR-99a exerts anti-metastasis through inhibiting myotubularin-related protein 3 expression in oral cancer. Oral Dis 20: e65-e75, 2014.

37. Turcatel G, Rubin N, El-Hashash A and Warburton D: MIR-99a and MIR-99b modulate TGF- $\beta$ induced epithelial to mesenchymal plasticity in normal murine mammary gland cells. PLoS One 7: e31032, 2012

38. Wang L, Chang L, Li Z, Gao Q, Cai D, Tian Y, Zeng L and Li M: miR-99a and -99b inhibit cervical cancer cell proliferation and invasion by targeting mTOR signaling pathway. Med Oncol 31: 934, 2014

39. Peruzzi F, Prisco M, Dews M, Salomoni P, Grassilli E, Romano G, Calabretta B and Baserga R: Multiple signaling pathways of the insulin-like growth factor 1 receptor in protection from apoptosis. Mol Cell Biol 19: 7203-7215, 1999.

40. Plowright EE, Li Z, Bergsagel PL, Chesi M, Barber DL, Branch DR, Hawley RG and Stewart AK: Ectopic expression of fibroblast growth factor receptor 3 promotes myeloma cell proliferation and prevents apoptosis. Blood 95: 992-998, 2000.

41. Castedo M, Ferri KF and Kroemer G: Mammalian target of rapamycin (mTOR): Pro- and anti-apoptotic. Cell Death Differ 9: 99-100, 2002

42. Catto JW, Miah S, Owen HC, Bryant H, Myers K, Dudziec E, Larré S, Milo M, Rehman I, Rosario DJ, et al: Distinct microRNA alterations characterize high- and low-grade bladder cancer. Cancer Res 69: 8472-8481, 2009. 
43. Wang $\mathrm{H}$, Li Q, Niu X, Wang G, Zheng S, Fu G and Wang Z: miR-143 inhibits bladder cancer cell proliferation and enhances their sensitivity to gemcitabine by repressing IGF-1R signaling. Oncol Lett 13: 435-440, 2017.

44. Park SJ, Lee TJ and Chang IH: Role of the mTOR pathway in the progression and recurrence of bladder cancer: An immunohistochemical tissue microarray study. Korean J Urol 52: 466-473, 2011.

45. Hammam O, Aboushousha T, El-Hindawi A, Khairy H, Khalil H, Kamel A, Akl M, Abdel-Hady A, Magdy M, Badawy M, et al: Expression of FGFR3 protein and gene amplification in urinary bladder lesions in relation to schistosomiasis. Open Access Maced J Med Sci 5: 160-166, 2017.

46. Tang L and Zhang Y: Mitochondria are the primary target in isothiocyanate-induced apoptosis in human bladder cancer cells. Mol Cancer Ther 4: 1250-1259, 2005.

47. Singh SV and Singh K: Cancer chemoprevention with dietary isothiocyanates mature for clinical translational research. Carcinogenesis 33: 1833-1842, 2012.

48. Bader AG: miR-34 - a microRNA replacement therapy is headed to the clinic. Front Genet 3: 120, 2012.

49. Zhang Y, Wang Z and Gemeinhart RA: Progress in microRNA delivery. J Control Release 172: 962-974, 2013.

50. Jiang SJ, Ye LY and Meng FH: Comparison of intravesical bacillus Calmette-Guerin and mitomycin $\mathrm{C}$ administration for non-muscle invasive bladder cancer: A meta-analysis and systematic review. Oncol Lett 11: 2751-2756, 2016.

51. Nogawa M, Yuasa T, Kimura S, Tanaka M, Kuroda J, Sato K, Yokota A, Segawa H, Toda Y, Kageyama S, et al: Intravesical administration of small interfering RNA targeting PLK-1 successfully prevents the growth of bladder cancer. J Clin Invest 115: 978-985, 2005.
52. Uchino K, Takeshita F, Takahashi RU, Kosaka N, Fujiwara K, Naruoka H, Sonoke S, Yano J, Sasaki H, Nozawa S, et al: Therapeutic effects of microRNA-582-5p and $-3 p$ on the inhibition of bladder cancer progression. Mol Ther 21: 610-619, 2013.

53. Inamoto $T$, Taniguchi $K$, Takahara $K$, Iwatsuki A, Takai $T$, Komura K, Yoshikawa Y, Uchimoto T, Saito K, Tanda N, et al: Intravesical administration of exogenous microRNA-145 as a therapy for mouse orthotopic human bladder cancer xenograft. Oncotarget 6: 21628-21635, 2015.

54. Lamy E, Scholtes C, Herz C and Mersch-Sundermann V: Pharmacokinetics and pharmacodynamics of isothiocyanates. Drug Metab Rev 43: 387-407, 2011.

55. Hadaschik BA, Black PC, Sea JC, Metwalli AR, Fazli L, Dinney CP, Gleave ME and So AI: A validated mouse model for orthotopic bladder cancer using transurethral tumour inoculation and bioluminescence imaging. BJU Int 100: 1377-1384, 2007.

56. Kota J, Chivukula RR, O'Donnell KA, Wentzel EA, Montgomery CL, Hwang HW, Chang TC, Vivekanandan P, Torbenson M, Clark KR, et al: Therapeutic microRNA delivery suppresses tumorigenesis in a murine liver cancer model. Cell 137: 1005-1017, 2009.

57. ToffaninS,Villanueva A andLlovetJM:miRNAdelivery: Emerging therapy for hepatocellular carcinoma. Gastroenterology 138: 1202-1204, 2010 